\title{
Cyanine Dyes: Fine Structures in Their Absorption Spectra
}

\author{
Anna Zarow and Yeung-gyo Shin \\ Department of Chemistry/Physics \\ Kean University \\ 1000 Morris Avenue \\ Union, New Jersey 07083 USA
}

Received: October 13, $2008 \quad$ Accepted: May 19, 2009

\begin{abstract}
Absorption spectra were studied for two series of 3 cyanine dyes with varying length of conjugated hydrocarbon chains. Fine sturctures in absortpiton spectra were analyzed to determine its concentraion dependence as the concentraion of dyes were changed from $10^{-8} \mathrm{M}$ to $10^{-4} \mathrm{M}$. In all 6 dyes studied, ratios of minor peaks to the major peak remained constant within the experiemntal error. These results indicate that the origin of the absorption fine structure is due to the electronic coupling, an intramolecular process, rather than the aggragates formation, an intermolecular process.
\end{abstract}

\section{INTRODUCTION}

Cyanine and related dyes are possible candidates in constructing efficient light harvesting devices for these molecules display very strong electronic absorption bands in the visible spectral range [1-3]. These dye molecules assume nearly planar geometry with abundant $\pi$-electrons which provides possibility of obtaining a well organized crystal structure. Such $\pi$-stacking interaction are common for many organic conductors and provide efficient pathways for shuttling electrons through the resulting macrostructures [4,5]. Therefore, understanding the spectral features of these dyes are essential in designing useful devices.

The "Particle in a Box" model is commonly introduced in undergraduate Physical Chemistry [6] and works reasonably well in predicting absorption maxima of conjugated $\pi$-systems: the maximum of electronic transition energy shifts toward lower energy as the length of conjugation or size of the box where the electron is trapped becomes larger. However, the actual absorption spectra of all 6 dyes studied exhibit well-defined fine structures with progression of 1100 to 1200 $\mathrm{cm}^{-1}$. This observation is not accordance with Particle in a Box estimation nor a state of the art computational method [7-9] which predict the absorption spectra of these dyes to be consist of a smooth single peak. One possible explanation of the caused for the splitting is attributed to the ability of the dye molecules to form dimmers or trimers as mentioned in "Experiments in Physical Chemistry". This explanation is very attractive one since resonance structure prevalent throughout the molecule implies extensive $\pi$-electron network that forces the molecule to be planar. More recently, vibronic coupling mechanism was used to explain the fine structures [10].

In this report, we would like to establish concentration dependence (or independence) of absorption band shape to provide better understanding and explanation of the origin of the fine-structure. Two families of Cyanine dyes were employed (Figure 1). Series / dyes include 1,1'-diethyl-2,2'-cyanine iodide (la, $\mathrm{n}=1)$, $1,1^{\prime}$-diethyl-2,2'-carbocyanine iodide $(I b, n=3)$ and 1,1'-dicarbodiethyl-2,2'-cyanine iodide $(I c, \mathrm{n}=5)$, where as the Series 2 dyes include $1,1^{\prime}$-diethyl-4,4'-cyanine iodide (Ila, $\mathrm{n}=1$ ), 1,1'-diethyl-4,4'-carbocyanine iodide ( $/ l b$, 
<smiles></smiles>

Series 1

Figure 1. Structures of Cyanine Dyes.

$\mathrm{n}=3)$ and 1,1'-diethyl-4,4'-dicarbocyanine iodide $(I / c, \mathrm{n}=5)$.

\section{EXPERIMENTAL}

\section{a. Chemicals}

All 6 cyanine dyes (99.9\% purity) as well as methanol (HPLC grade) were purchased from Aldrich Chemical Co. and used as received.

\section{b. Acquisition and Analysis of Spectra}

Absorption spectra were acquired in methanol using Milton Roy Spectronic 3000 Array spectrometer equipped with a diode array detector. Since all cyanine dyes under investigation has molar absorptivity in the range of $10^{5} \mathrm{M}^{-1} \mathrm{~cm}^{-1}$, stock solution of about $10^{-3} \mathrm{M}$ was prepared in methanol then this stock solution was diluted down to $10^{-8}$ M. Absorbance of any given spectrum was kept under 1 , though the concentration of the dyes was changed up to 10000 fold.

For dilute samples, cells with path length of $1 \mathrm{~cm}$ or $2 \mathrm{~mm}$ were used. For every scan, a reference spectrum was acquired using pure methanol before taking the spectrum of the solution. For the stock solution, a micro cell was constructed using two slide-glasses and Kapton ${ }^{\circledR}$ spacer with different thickness $(25,50$ or $75 \mu \mathrm{m})$. The reference spectrum was acquired using an empty micro cell then the stock solution was added to the identical cell. Though this kind of micro cell is not as optically precise as the ones on the market, it afforded us to look into concentrated solutions with absorbance of 100 or more in a traditional $1-\mathrm{cm}$ cell.

Spectra were initially acquired as the function of wavelength then later

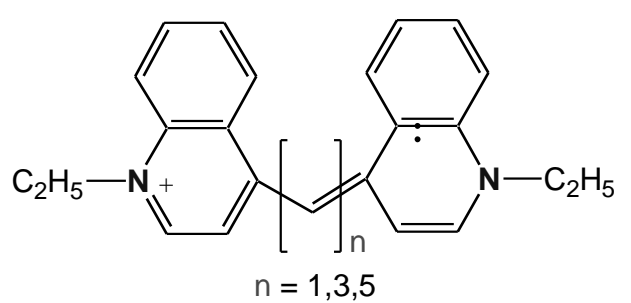

Series 2 converted to spectra as the function of wavenumber. The resulting spectra were analyzed utilizing multi-peak fitting package provided in IGOR Pro (v. 5.01) program. Individual peaks were assumed to be Gaussian in nature with varying but similar peak width. The number of peaks included in the fitting was determined by examining the residual values whose maximum values routinely became less then $1 \%$ of the major peak height.

\section{DISCUSSION}

Cyanine dyes examined in this study contains conjugated systems with alternating double bonds. Delocalized $\pi-$ bonds present in the molecule provide their valence electrons that occupy molecular orbitals and are able to resonate or travel along the conjugated chain in the molecule. Depending on the length of the conjugated chain, absorption bands due to the electronic transitions of $\pi$-electrons along the molecule's chain appear in the different energy of the UV-Visible spectral region; approximately 400 to $800 \mathrm{~nm},(25000$ to $\left.12500 \mathrm{~cm}^{-1}\right)$. Behavior of these delocalized electrons along the molecule's chain can be represented as "a particle in a one dimensional box". This simple theory predicts a well-isolated sinlge peak at the postion determined by following equation.

$$
\Delta E=\frac{h^{2}}{8 m L^{2}}(N+1)
$$

where $\mathrm{h}$ is Plank's constant; $\mathrm{m}$ is the mass of electron; $L$ is the length of conjugation, and $\mathrm{N}$ is the number of electrons in the conjugated chain. Use of computational methods [7,8], such as density functional theory [10] or semi-empirical INDO method 
[9] also predicts a well-isolated single peak for the transition.

Spectrum of Dye la in Figure 2 clearly shows at least three distinct peaks with progression of $\sim 1200 \mathrm{~cm}^{-1}$. To explain this fine structure, a popular textbook for undergraduate physical chemistry laboratory introduces mechanism of "Dimerization" [6]. Since the cyanine dyes are predicted to be planar molecules owing to the extensive conjugation throughout the molecules, it is possible to stack them on top of each other $[4,5]$. Upon the formation of dimer, molecular orbitals of adjoining dyes will interact and cause them to split into bonding and antibonding orbitals, thus creating a new peak. Similar explanation was successfully employed for the related family of dyes, phthalocyanines. Since the planar configuration of the dye will be conserved in the dimer, it is conceivable to imagine a timer where three dye molecules are stacked to explain the third peak clearly identifiable in the spectrum.

The Series II dyes where the bridging group is at para-position to the $\mathrm{N}$ atoms that defines the limits of the box show less pronounced higher energy peaks than the Series / dyes. Series I/ dyes are not as planar as the Series I dyes, since the position of the bridging group forces the bulky naphtyl groups to be close each other. Therefore, Series II dyes will be harder to stack than the Series / dyes, resulting in a lower concentration of dimers or less pronounced minor peaks.

This "dimerization" mechanism rests upon the favorable interaction between two dye molecules with an association constant, $\mathrm{K}_{\mathrm{a}}$. As two dye molecules stack on top of each other, the $\pi$-cloud of a molecule will interact with the adjacent molecule and their molecular orbitals will split into bonding and anti-bonding pair. In the simplest estimation the splitting would be $2 \beta$, where $\beta$ is stabilization. The resulting transition energy will be red shifted by $2 \beta$ for dimers, $4 \beta$ for trimer, etc. Since the spectra show nice progression of peaks with $\Delta v$ of $1100 \mathrm{~cm}^{-1}$ to $1300 \mathrm{~cm}^{-1}, \beta$ value is estimated to be $\sim 600 \mathrm{~cm}^{-1}$ which is in accordance with reported values [11].

If the dimerization mechanism is in operation, a dynamic chemical equilibrium will be established between monomers and

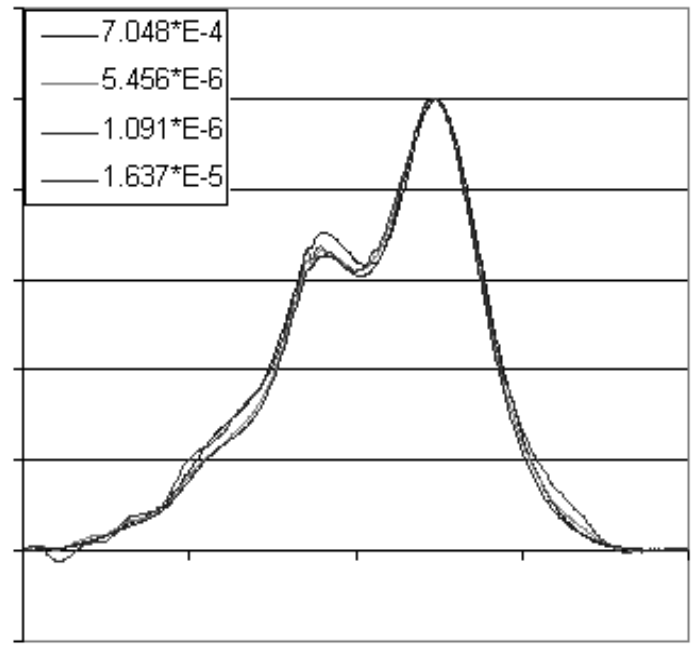

Figure 2. Overlay of absorption spectra of the dye $1 \mathrm{a}$ in 4 different concentrations.

dimer and possibly beyond. According to LeChatelier's principle, by adding monomers, or increasing the concentration of dyes, one should be able to shift the equilibrium to dimer, or vice versa. Thus the relative concentration of the dimer is predicted to increase as the formal concentration of the dye increases. To confirm this possibility, concentration dependence of the peak profile has to be performed to support.

Absorption spectra were acquired as the concentration of the dyes was changed from $10^{-8} \mathrm{M}$ to $10^{-3} \mathrm{M}$. Overlay of 4 normalized spectra (Figure 2) in wide range of concentration shows nearly constant absorption profile. This observation is further confirmed by deconvoluting the absorption profile using IGOR Pro program into several Gaussian peaks (Figure 3, Tables 1 and 2). Peak widths of all peaks in the same spectra were set to be the same for the initial fitting, and then the peak width restriction was lifted for the final fitting. All dyes require more than 3 Gaussian peaks to properly represent the absorption profile. Energy difference between peaks was 1100 to $1200 \mathrm{~cm}^{-1}$ in all 6 dyes followed by the much smaller gap between first two fitted peaks with the exception of Ila. This smaller, first energy gap is dependent on dyes ranging from 120 $\mathrm{cm}^{-1}$ for la to $900 \mathrm{~cm}^{-1}$ of la. Series of structural optimizations of each dyes using PC Spartan Pro (PM3) found several other viable structures through cis-trans isomerization of bridging $\mathrm{CH}_{2}$ groups. The 


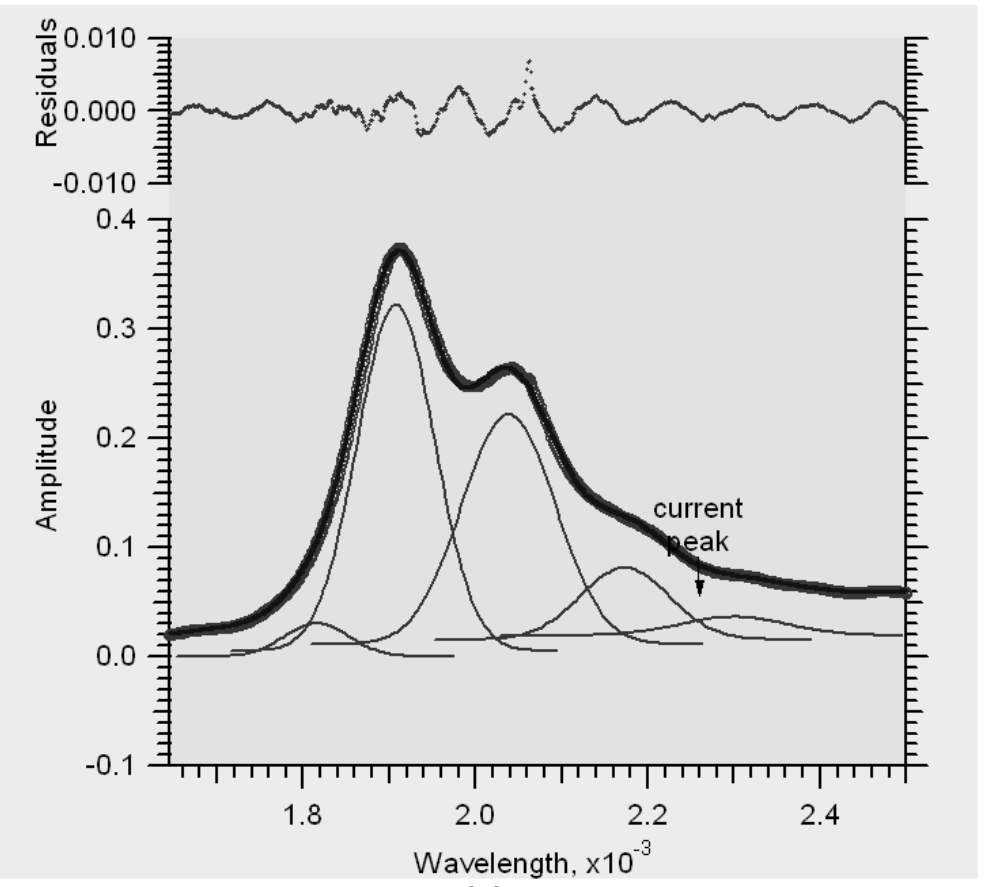

(a)

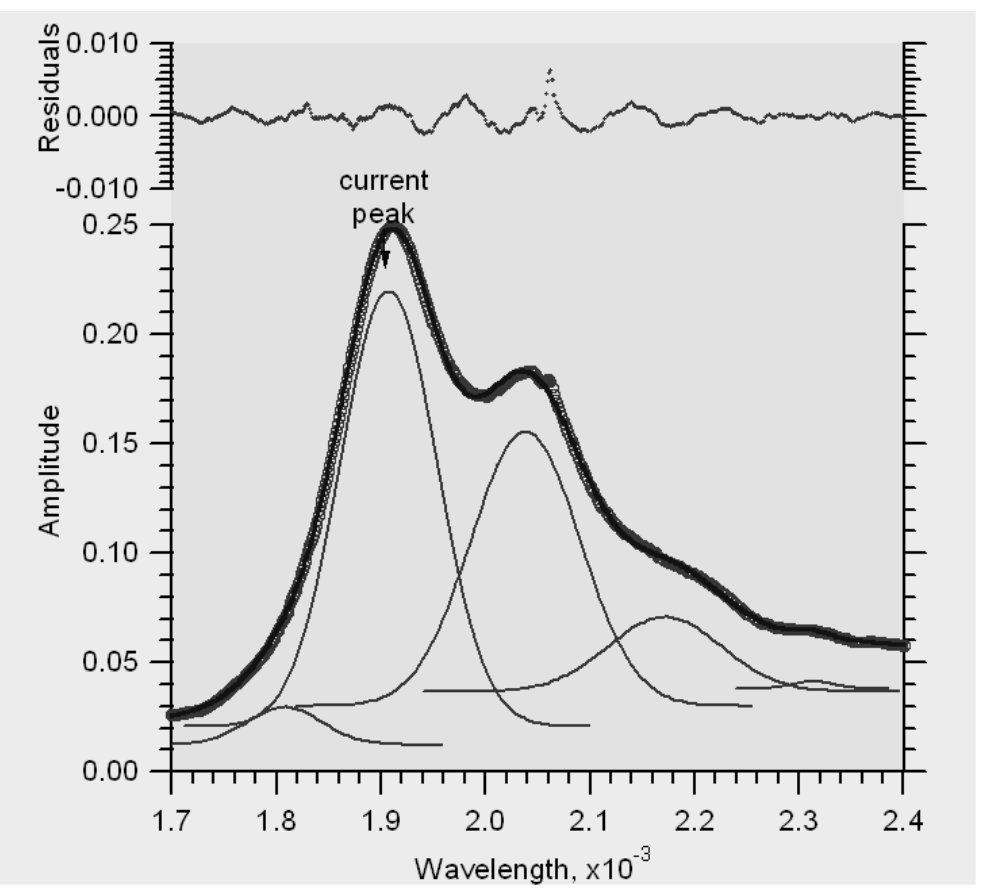

(b)

Figure 3. Fine structure in the absorption spectra of Dye 1a with concentrations of (a) $4.36 \times 10^{-6}$

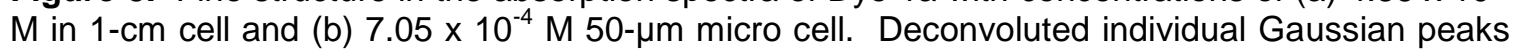
using IGOR Pro are indicated.

heats of formation for these structures were clustered within 4 to $5 \mathrm{~kJ} / \mathrm{mol}$ indicating population of other structures can not be ignored. Computational study of electronic transition energy as a function of strucural variation is being performed and will be reported elsewhere.

Deviation of all peak positions were less then $1 \%$, whereas the deviation of peak intensity for the second highest peak is 
Table 1. Deconvolution of Absorption Peak: Series I

\begin{tabular}{ccccc}
\hline Dye & Position / kK & $\sigma_{\text {position }} * / \mathrm{kK}$ & $\begin{array}{c}\text { Relative } \\
\text { Intensity }\end{array}$ & $\sigma_{\text {intensity }}{ }^{*}$ \\
\hline \hline Ia & 18.2 & 0.11 & 0.11 & 0.04 \\
& 19.1 & 0.04 & 1.00 & 0.02 \\
& 20.4 & 0.17 & 0.81 & 0.08 \\
& 21.7 & 0.06 & 0.24 & 0.09 \\
Ib & 22.8 & 0.13 & 0.12 & 0.02 \\
\hline Ic & 16.5 & 0.005 & 0.37 & 0.09 \\
& 16.6 & 0.001 & 1.00 & 0.05 \\
& 17.8 & 0.001 & 0.60 & 0.02 \\
& 19.0 & 0.004 & 0.20 & 0.05 \\
\hline \hline
\end{tabular}

Table 2. Deconvolution of Absorption Peak: Series II

\begin{tabular}{ccccc}
\hline Dye & Position $/ \mathrm{kK}$ & $\sigma_{\text {position }} * / \mathrm{kK}$ & Relative Intensity & $\sigma_{\text {intensity }}{ }^{*}$ \\
\hline \hline IIa & 16.9 & 0.02 & 1.00 & 0.02 \\
& 18.1 & 0.06 & 0.34 & 0.05 \\
& 19.4 & 0.2 & 0.04 & 0.02 \\
\hline IIb & 14.1 & 0.006 & 1.000 & 0.01 \\
& 14.3 & 0.01 & 0.762 & 0.02 \\
& 15.4 & 0.02 & 0.350 & 0.02 \\
IIc & 16.4 & 0.1 & 0.153 & 0.07 \\
& 12.3 & 0.003 & 0.60 & 0.08 \\
& 12.5 & 0.02 & 1.00 & 0.01 \\
& 13.5 & 0.006 & 0.66 & 0.06 \\
\hline
\end{tabular}

* Standard deviation was calculated using individual relative intensity as well as using error propagation based on the standard deviation of individual fitting. The larger of two is reported.

about $5 \%$ and increases as the relative peak intensity deceases. It is noteworthy that the deviation in intensity of each concentration is completely random with respect to the dye concentration. If there is any correlation, it is with the absolute absorbance of the sample, i.e. the smaller the absolute absorbance, the larger the intensity deviation. This may be rectified if many cells with different path-lengths were employed so that all absorption spectra will have similar absorbance.
Fine structure in the absorption spectra of Dye 1a with concentrations of Observation of the identical peak profile upon the variation of concentration up to 10000 fold poses a serious problem to the "dimerization" mechanism. First, the idea of establishing a dynamic equilibrium between monomers and dimers cannot be justified. If the equilibrium is disturbed by adding more monomer, i.e. the concentration of the dye is increased; concentration of the dimer should be also increased not linearly but proportional to the $\mathrm{C}^{2}$ and dimer/monomer 


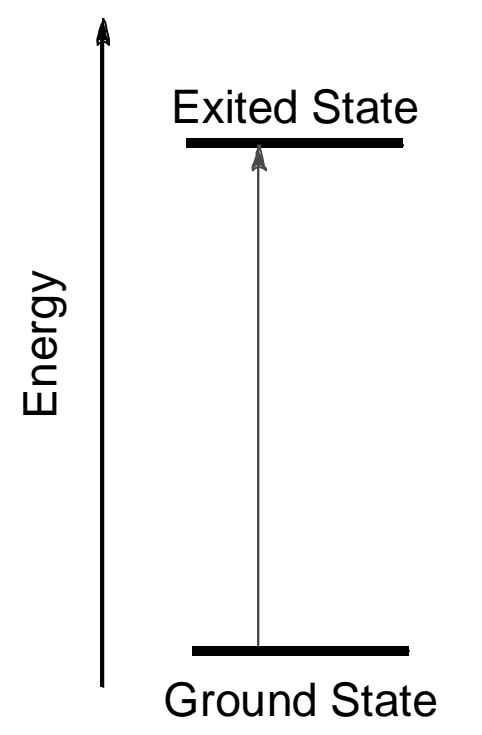

particle in a box

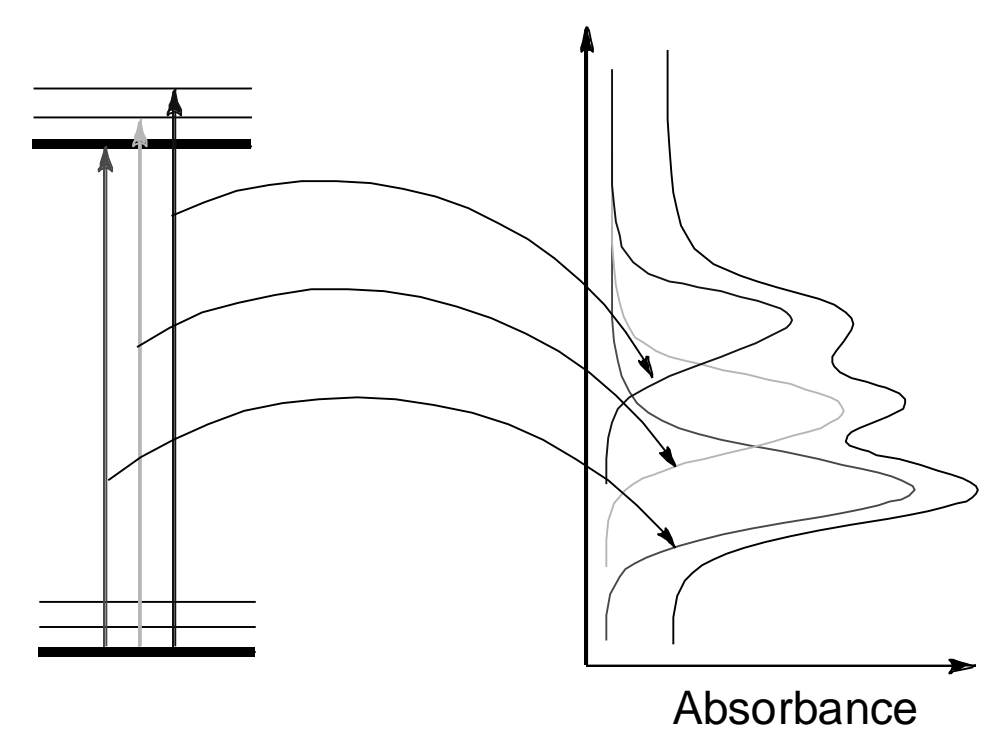

with vibronic coupling

Figure 4. Schematic diagram of the fine structure originating from the vibronic coupling mechanism. For clarity, only three vibrational levels are included.

ratio should be approximately proportional to C. Second, up to 5 peaks are needed to properly fit the absorption profile. Concentration of trimer ( 3 molecules in a unit) should be much smaller than that of dimer and any higher order aggregates should be scarce at best. In addition, concentrations of these higher order aggregates will be even more sensitive to the concentration of the dye. Third, deconvoluted Gaussian peaks have relatively constant peak width. As the aggregate size becomes larger, interaction among them will be less defined. Such illdefined interaction will produce less defined energy level, thus causing the absorption peak width to increase for the higher order aggregates represented by minor peaks. Fourth, minor peaks appear on the higher energy side. If the dimer is formed, splitting of HOMO and LUMO will each produce bonding-antibonding orbitals. Therefore, the resulting electronic transition should be from the antibonding component of HOMO to bonding component of LUMO, which will have a lower energy than that of the monomer.

Based on the observation, one can conclude that the mechanism of producing the fine structures in the absorption spectra of cyanine dyes should be an intramolecular mechanism. In a different cyanine dyes, presence of two different isomers, i.e. cisand trans-configuration of two bulky end groups was proposed as one such mechanism [10]. Our study found that cistrans isomerization can be considered for explaining a part of splitting; it can not explain the complete fine structure.

To this end, "vibronic coupling", an intramolecular mechanism, seems to be the choice of mechanism for generating the finestructure of the absorption spectra. By coupling a vibrational mode to an electronic state either occupied or unoccupied, it is possible to access higher level vibrational states as well as the vibrational ground state (Figure 4). In room temperature, most of the higher level vibrational states are not populated, because typical vibrational energy $\left(\sim 1200 \mathrm{~cm}^{-1}\right.$ in our case) is much higher than energy represented by the room temperature $\left(\sim 200 \mathrm{~cm}^{-1}\right)$. Therefore, the electronic transition for absorption is from the vibrational ground state nestled in the electronic ground state to the vibrational ground or exited state nestled in the electronic excited state. In other words, the absorption spectra will probe the vibrational mode of the excited state. If emission 
spectrum has similar fine structure, one can probe vibrational modes of the molecule in the ground state.

\section{CONCLUSIONS}

Spectral profiles of absorption spectra of cyanine dyes do not change within the concentraion range studied $\left(10^{-3}\right.$ to $10^{-8}$ ). The mechanism of producing the fine structure is not intermolecular but intramolecular mechanism, thus the dimerization or aggragation cannot explain the fine structure. Presence of multiple peaks in la where bridge has only one $\mathrm{CH}_{2}$ group, precludes cis-trans isomerization (an intramolecular mechanism) as a possible cause of entire fine structure. Only the vibronic coupling can explain independence of the spectral profile to concentration, presence of multiple peaks, nearly constant peak width, and their energy splitting.

\section{ACKNOWLEDGEMENT}

Kean University: Student partnering with Faculty: summer research program, 2004

\section{REFERENCES}

1. Chen, X.; Guo, J.; Peng, X; Guo, M.; Xu, Y.; Shi, L.; Liang, C; Wang, L.; Gao, Y.; Sun, S.; Cai, S. J. Photochem. \& Photobiol. A: Chemistry, 2005, 171, 235-240.
2. Matsui, M.; Hashimoto, Y.; Funabiki, K.; Jin, J.-Y.; Yoshida, T.; Minoura, H. Synthetic Metals, 2005, 148, 147-153.

3. Kassab, K. J. Photochem. \& Photobiol. B: Biology, 2002, 68, 15-22.

4. Xiang, J.; Chen, C.; Chen, Z.; Yan, Y.; Ai, X.; Liu, Y.; Xu. G. J. Colloid and Interface Science, 2002, 254, 195-199.

5. Bujdák, J.; Iyi, N; Fujita, T. Colloids and Surfaces A: Physicochemical and Engineering Aspects, 2002, 1-3, 207214.

6. Garland, C. W.; Nibler, J. W.; Shoemaker, D. P., "Experiments in Physical Chemistry", $7^{\text {th }}$ ed. McGraw Hill, New York, 2009, 393-398.

7. Yuan, S.; Chen, Z. J. Mol. Structure: Theochem, 2005, 717, 81-84.

8. Anderson, B. D., J. Chem. Ed., 1997, 74, 985.

9. Zambell, C.; Shin, Y.-g. K. "Conjugated Dyes: Particle in a Box vs. Computation" The $9^{\text {th }}$ National Conference of the Council on Undergraduate Research, New London, CT, June, 2002.

10. Champagne, B.; Guillaume, $\quad M$,; Zutterman, F., Chemical Physics Letters, 2006, 425, 105-109.

11. Mao, L.S.; Wang, Y.L.; Hu, X.C., J. Phys. CHem. B, 2003, 107, 3963-3971.

12. Sahyun, M. R. V.; Blair, J. T. J. Photochem \& Photobiol A: Chemistry, 1997, 104, 179-187.

\section{Kean University • www.kean.edu}

KE A N

UNIVER S I T Y

Life. Enroll Now.

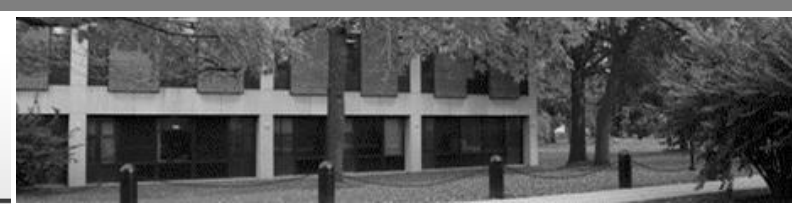

Founded in 1855, Kean University has grown to become one of New Jersey's largest institutions of higher learning. In 1958, Kean moved from Newark to Union and currently occupies over 150 acres in Union and Hillside Townships. Kean was granted university status on September 26, 1997. While maintaining its significant role in the training of teachers, Kean has become a comprehensive institution offering 48 undergraduate and 28 graduate degree programs serving some 13,000 students. Across four undergraduate colleges and the Nathan Weiss Graduate College, Kean's academic programs cover an exceptional range of disciplines. 


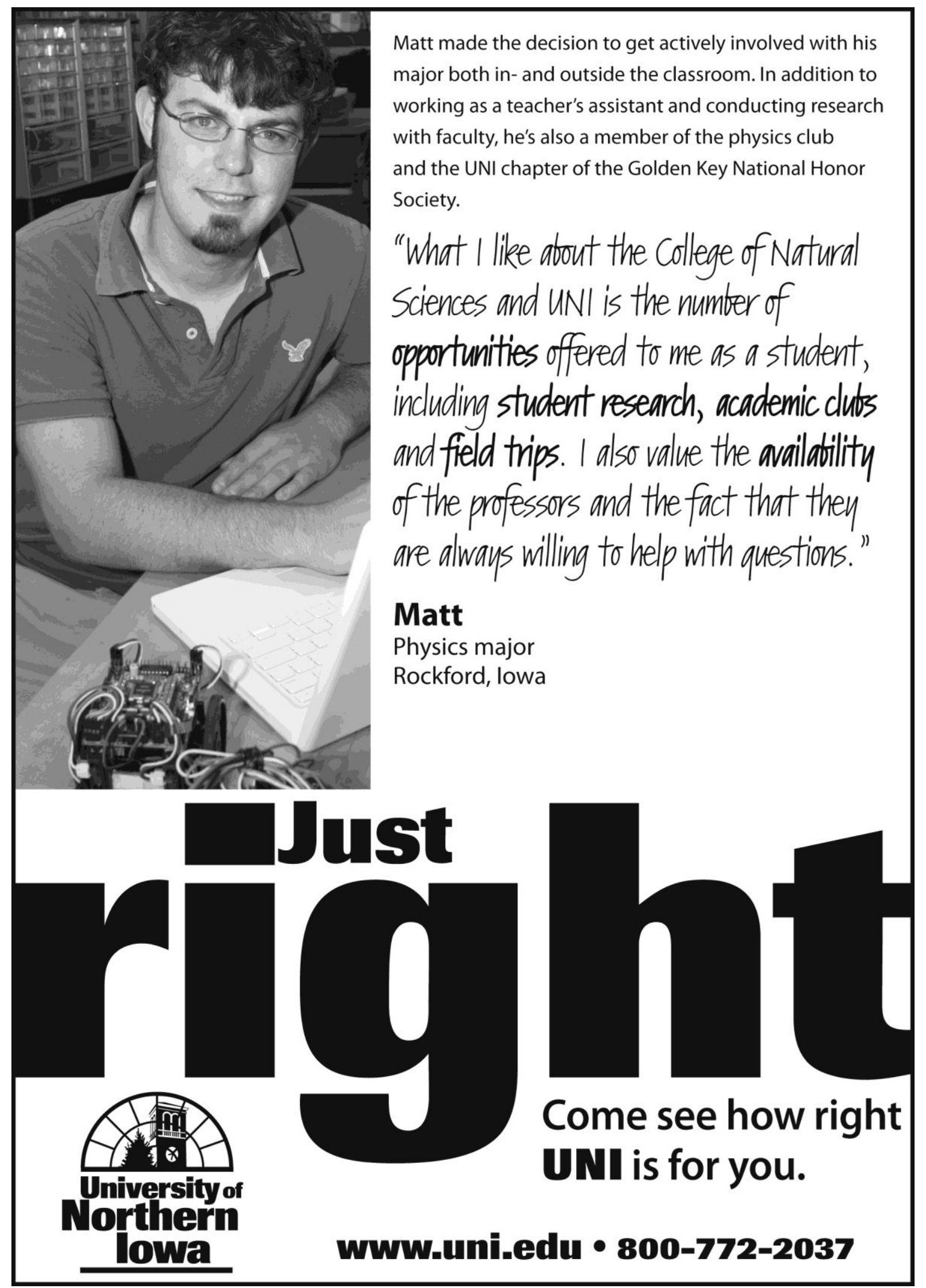

